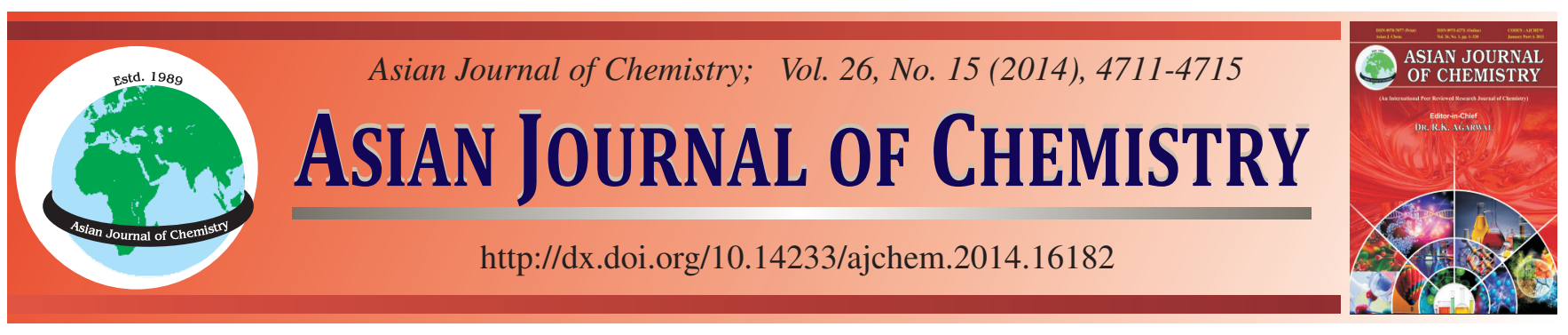

\title{
Molecular Interactions in Binary Liquid Mixtures of Cyclohexanone with Morpholine, Bromobenzene and Anisole at 308.15 K and 318.15 K
}

\section{A. Rose Venis ${ }^{1, *}$ and X. Rosario RAJKUMAR ${ }^{2}$}

${ }^{1}$ Department of Chemistry, St. Joseph's College (Autonomous), Tiruchirappalli-620 002, India ${ }^{2}$ Oxford Engineering College, Tiruchirappalli-620 009, India

*Corresponding author: E-mail: rosevenis@gmail.com

\begin{abstract}
Densities, viscosities and ultrasonic velocities have been measured for the binary mixtures of cyclohexanone with morpholine, bromobenzene and anisole at $308.15 \mathrm{~K}$ and $318.15 \mathrm{~K}$ over the entire range of mole fraction. Thermodynamic parameters like excess volume, adiabatic compressibility, free volume, linear free energy, acoustic impedance and viscosity are calculated. From these values the excess properties are also calculated. The deviations of the liquid mixture from ideality have been explained based on the nature molecular interactions between unlike molecules.
\end{abstract}

Keywords: Density, Viscosity, Ultrasonic velocity, Adiabatic compressibility, Acoustic impedance.

\section{INTRODUCTION}

Physical and transport properties of binary liquid mixtures $^{1-5}$ are of great importance in industrial process. Cyclohexanone $e^{6-9}$ is a cyclic ketone which finds many application in organic synthesis and industrial applications. Similarly morpholine $e^{10-12}$, bromobenzene ${ }^{13-15}$ and anisole ${ }^{16-18}$ are of great importance in organic process. This lead to study the properties of binary liquid mixtures of these liquids based on the nature of interactions between the mixing liquids. In the present study the nature of interactions has been studied based on the measurement of densities, viscosities and ultrasonic velocities of all pure liquids and their constituent binaries at $308.15 \mathrm{~K}$ and $318.15 \mathrm{~K}$. From these values excess volume, adiabatic compressibility, linear free energy, acoustic impedance, free volume and viscosities were calculated. Excess values for all these thermodynamic parameters were calculated and fitted to Redlich-Kister polynomial to derive the binary coefficients and standard deviations between the calculated and fit parameters.

\section{EXPERIMENTAL}

Cyclohexanone (Merck, Mumbai), morpholine (Merck, Mumbai), bromo benzene (Nice chemicals, Cochin) and anisole (Nice chemicals, Cochin), all AnalaR grades ${ }^{19}$ were dried using suitable drying agents and distilled based on standard methods. Binary liquid mixtures of various compositions were prepared by mixing measured amount of pure liquids in air tight stopper bottles of $50 \mathrm{~mL}$ capacity. Densities of pure liquids and liquid mixtures were measured by relative density method using $10 \mathrm{~mL}$ relative density bottle with an accuracy of $\pm 0.001 \mathrm{kgm}^{-3}$. Viscosities of all pure and liquid mixtures were measured using Ostwald viscometer of $10 \mathrm{~mL}$ capacity with an accuracy of $\pm 0.001 \mathrm{cP}$. Ultrasonic velocities of pure and liquid mixtures were measured by a single crystal variable path interferometer (Mittal Enterprises, New Delhi, model F-80) at a frequency of $2 \mathrm{MHz}$ with an accuracy of \pm $0.02 \%$. All the measurements were made at both $308.15 \mathrm{~K}$ and $318.15 \mathrm{~K}$ with the help of a digital thermostat with a temperature accuracy of $\pm 0.01 \mathrm{~K}$.

\section{RESULTS AND DISCUSSION}

The experimental densities $(\rho)$, viscosity $(\eta)$ and ultrasonic velocity $(\mathrm{u})$ of the pure liquids and binary systems are presented in Table-1a and $1 \mathrm{~b}, 2 \mathrm{a}$ and $2 \mathrm{~b}$ and $3 \mathrm{a}$ and $3 \mathrm{~b}$.

Adiabatic compressibility (Ks) has been calculated from Laplace's equation $^{20}$

$$
\mathrm{Ks}=1 / \rho \mathrm{u}^{2}
$$

in which $\rho$ and $u$ are density and ultrasonic velocity in liquid, respectively.

Acoustic impedance $(Z)$ has been calculated by the relation $^{21}$

$$
\mathrm{Z}=\mathrm{u} \rho
$$

Linear free energy has been calculated by Jacobson's relation $^{22}$

$$
\mathrm{L}_{\mathrm{f}}=\mathrm{K} / \mathrm{u} \rho^{1 / 2}
$$


TABLE-1A

THERMODYNAMIC EXCESS PARAMETERS FOR THE BINARY MIXTURE MORPHOLINE (1)-CYCLOHEXANONE (2) 308.15 K

\begin{tabular}{|c|c|c|c|c|c|c|c|c|c|}
\hline $\mathrm{x}_{1}$ & $\begin{array}{c}\rho \times 10^{3} \\
\left(\mathrm{Kg} \mathrm{m}^{-3}\right)\end{array}$ & $\begin{array}{l}\mathrm{V}^{\mathrm{E}} \times 10^{3} \\
\left(\mathrm{~m}^{3} \mathrm{~mol}^{-1}\right)\end{array}$ & $\begin{array}{c}\mathrm{U} \\
\left(\mathrm{m} \mathrm{s}^{-1}\right)\end{array}$ & $\varphi_{1}$ & $\begin{array}{l}\Delta \mathrm{Ks}^{-1} \\
\mathrm{~T} \mathrm{~Pa}^{-1}\end{array}$ & $\Delta \eta \mathrm{cP}$ & $\Delta \mathrm{L}_{\mathrm{f}} \times 10^{5} \mathrm{~m}$ & $\begin{array}{c}\Delta \mathrm{Z} \times 10^{3} \\
\left(\mathrm{Kg} \mathrm{m}^{-2} \mathrm{~s}^{-1}\right)\end{array}$ & $\begin{array}{l}\Delta \mathrm{V}_{\mathrm{f}} \times 10^{-8} \\
\left(\mathrm{~m}^{3} \mathrm{~mol}^{-1}\right)\end{array}$ \\
\hline 0.0000 & 0.9312 & 0.0000 & 1358 & 0.0000 & 0.0000 & 0.0000 & 0.0000 & 0.0000 & 0.0000 \\
\hline 0.0985 & 0.9360 & -0.0197 & 1367 & 0.0940 & -1.1086 & 0.2560 & -0.4316 & 2.5217 & -1.5366 \\
\hline 0.1980 & 0.9412 & -0.0599 & 1371 & 0.1717 & -1.5704 & 0.2651 & -0.3149 & 0.8421 & -1.5530 \\
\hline 0.2934 & 0.9464 & -0.1037 & 1376 & 0.2584 & -2.8118 & 0.2764 & -0.3392 & 0.6821 & -1.5525 \\
\hline 0.3958 & 0.9522 & -0.1524 & 1382 & 0.3547 & -4.4096 & 0.2863 & -0.4393 & 1.4583 & -1.5195 \\
\hline 0.4934 & 0.9580 & -0.2062 & 1388 & 0.4498 & -5.9357 & 0.2988 & -0.5729 & 2.9115 & -1.4807 \\
\hline 0.5903 & 0.9639 & -0.2633 & 1392 & 0.5473 & -5.6463 & 0.3123 & -0.4966 & 2.7391 & -1.4403 \\
\hline 0.6987 & 0.9705 & -0.2811 & 1396 & 0.6606 & -4.5568 & 0.3229 & -0.3506 & 2.1333 & -1.3647 \\
\hline 0.7998 & 0.9762 & -0.2418 & 1400 & 0.7702 & -3.1173 & 0.2924 & -0.1992 & 1.2506 & -1.1609 \\
\hline 0.8944 & 0.9812 & -0.1567 & 1404 & 0.8766 & -1.4323 & 0.2631 & -0.0518 & 0.2471 & -0.9652 \\
\hline 1.0000 & 0.9863 & 0.0000 & 1410 & 1.0000 & 0.0000 & 0.0000 & 0.0000 & 0.0000 & 0.0000 \\
\hline
\end{tabular}

TABLE-1B

THERMODYNAMIC EXCESS PARAMETERS FOR THE BINARY MIXTURE MORPHOLINE (1)-CYCLOHEXANONE (2) 318.15 K

\begin{tabular}{|c|c|c|c|c|c|c|c|c|c|}
\hline $\mathrm{x}_{1}$ & $\begin{array}{c}\rho \times 10^{3} \\
\left(\mathrm{Kg} \mathrm{m}^{-3}\right) \\
\end{array}$ & $\begin{array}{l}\mathrm{V}^{\mathrm{E}} \times 10^{3} \\
\left(\mathrm{~m}^{3} \mathrm{~mol}^{-1}\right) \\
\end{array}$ & $\begin{array}{c}\mathrm{U} \\
\left(\mathrm{m} \mathrm{s}^{-1}\right) \\
\end{array}$ & $\varphi_{1}$ & $\begin{array}{c}\Delta \mathrm{Ks} \\
\mathrm{T} \mathrm{Pa}^{-1}\end{array}$ & $\Delta \eta \mathrm{cP}$ & $\Delta \mathrm{L}_{\mathrm{f}} \times 10^{5} \mathrm{~m}$ & $\begin{array}{c}\Delta \mathrm{Z} \times 10^{3} \\
\left(\mathrm{Kg} \mathrm{m}^{-2} \mathrm{~s}^{-1}\right) \\
\end{array}$ & $\begin{array}{l}\Delta \mathrm{V}_{\mathrm{f}} \times 10^{-8} \\
\left(\mathrm{~m}^{3} \mathrm{~mol}^{-1}\right) \\
\end{array}$ \\
\hline 0.0000 & 0.9234 & 0.0000 & 1275 & 0.0000 & 0.0000 & 0.0000 & 0.0000 & 0.0000 & 0.0000 \\
\hline 0.0985 & 0.9285 & -0.0703 & 1308 & 0.0940 & -22.0669 & 0.2631 & -2.8954 & 19.5768 & -2.0050 \\
\hline 0.1980 & 0.9339 & -0.1488 & 1334 & 0.1717 & -38.3674 & 0.2550 & -4.7389 & 33.1706 & -1.7756 \\
\hline 0.2934 & 0.9391 & -0.2079 & 1355 & 0.2584 & -48.7308 & 0.2295 & -5.9277 & 42.8304 & -1.4768 \\
\hline 0.3958 & 0.9450 & -0.2836 & 1372 & 0.3547 & -53.9102 & 0.2216 & -6.5105 & 48.6241 & -1.3244 \\
\hline 0.4934 & 0.9508 & -0.3528 & 1379 & 0.4498 & -50.5353 & 0.1965 & -6.0154 & 45.8304 & -1.1411 \\
\hline 0.5903 & 0.9566 & -0.4087 & 1380 & 0.5473 & -41.9114 & 0.1908 & -4.8654 & 37.5615 & -1.1117 \\
\hline 0.6987 & 0.9631 & -0.4399 & 1382 & 0.6606 & -32.3185 & 0.1822 & -3.6626 & 29.0697 & -1.0460 \\
\hline 0.7998 & 0.9681 & -0.3488 & 1384 & 0.7702 & -22.3091 & 0.1739 & -2.4707 & 19.8949 & -0.9698 \\
\hline 0.8944 & 0.9723 & -0.2037 & 1386 & 0.8766 & -12.2355 & 0.1472 & -1.3279 & 10.7856 & -0.7971 \\
\hline 1.0000 & 0.9767 & 0.0000 & 1388 & 1.0000 & 0.0000 & 0.0000 & 0.0000 & 0.0000 & 0.0000 \\
\hline
\end{tabular}

TABLE-2A

THERMODYNAMIC EXCESS PARAMETERS FOR THE BINARY MIXTURE BROMOBENZENE (1)-CYCLOHEXANONE (2) 308.15 K

\begin{tabular}{|c|c|c|c|c|c|c|c|c|c|}
\hline $\mathrm{X}_{1}$ & $\begin{array}{c}\rho \times 10^{3} \\
\left(\mathrm{Kg} \mathrm{m}^{-3}\right)\end{array}$ & $\begin{array}{l}\mathrm{V}^{\mathrm{E}} \times 10^{3} \\
\left(\mathrm{~m}^{3} \mathrm{~mol}^{-1}\right) \\
\end{array}$ & $\begin{array}{c}\mathrm{U} \\
\left(\mathrm{m} \mathrm{s}^{-1}\right)\end{array}$ & $\varphi_{1}$ & $\begin{array}{c}\Delta \mathrm{Ks} \\
\mathrm{T} \mathrm{Pa}^{-1}\end{array}$ & $\Delta \eta \mathrm{cP}$ & $\Delta \mathrm{L}_{\mathrm{f}} \times 10^{6} \mathrm{~m}$ & $\begin{array}{c}\Delta \mathrm{Z} \times 10^{3} \\
\left(\mathrm{Kg} \mathrm{m}^{-2} \mathrm{~s}^{-1}\right)\end{array}$ & $\begin{array}{l}\Delta \mathrm{V}_{\mathrm{f}} \times 10^{-8} \\
\left(\mathrm{~m}^{-3} \mathrm{~mol}^{-1}\right)\end{array}$ \\
\hline 0.0000 & 0.9312 & 0.0000 & 1358 & 0.0000 & 0.0000 & 0.0000 & 0.0000 & 0.0000 & 0.0000 \\
\hline 0.0954 & 0.9864 & -0.3095 & 1340 & 0.0963 & -10.5349 & 0.0768 & -0.1845 & 20.6010 & -1.4449 \\
\hline 0.1935 & 1.0420 & -0.4881 & 1320 & 0.1951 & -20.7921 & -0.0192 & -0.3182 & 36.6241 & -1.3364 \\
\hline 0.2908 & 1.0947 & -0.4236 & 1300 & 0.2930 & -27.5345 & -0.0509 & -0.4060 & 46.9600 & -1.4656 \\
\hline 0.3939 & 1.1483 & -0.1667 & 1260 & 0.3964 & -15.8133 & -0.0705 & -0.2422 & 31.1486 & -1.7634 \\
\hline 0.4931 & 1.1996 & -0.0733 & 1222 & 0.4957 & -2.5395 & -0.1134 & -0.0584 & 12.1387 & -1.2863 \\
\hline 0.5956 & 1.2517 & -0.3681 & 1188 & 0.5981 & 8.9657 & -0.1110 & 0.1010 & -6.0821 & -1.2644 \\
\hline 0.6908 & 1.2983 & -0.7597 & 1160 & 0.6930 & 18.7215 & -0.0663 & 0.2361 & -23.6020 & -2.0927 \\
\hline 0.8239 & 1.3670 & -0.9710 & 1138 & 0.8254 & 15.9346 & -0.0516 & 0.2063 & -25.0544 & -1.2130 \\
\hline 0.9190 & 1.4220 & -0.6535 & 1126 & 0.9198 & 9.1144 & -0.0165 & 0.1197 & -16.0183 & -0.9878 \\
\hline 1.0000 & 1.4743 & 0.0000 & 1118 & 1.0000 & 0.0000 & 0.0000 & 0.0000 & 0.0000 & 0.0000 \\
\hline
\end{tabular}

TABLE-2B

THERMODYNAMIC EXCESS PARAMETERS FOR THE BINARY MIXTURE BROMOBENZENE (1)-CYCLOHEXANONE (2) 318.15 K

\begin{tabular}{|c|c|c|c|c|c|c|c|c|c|}
\hline $\mathrm{x}_{1}$ & $\begin{array}{c}\rho \times 10^{3} \\
\left(\mathrm{Kg} \mathrm{m}^{-3}\right)\end{array}$ & $\begin{array}{l}\mathrm{V}^{\mathrm{E}} \times 10^{3} \\
\left(\mathrm{~m}^{3} \mathrm{~mol}^{-1}\right)\end{array}$ & $\begin{array}{c}\mathrm{U} \\
\left(\mathrm{m} \mathrm{s}^{-1}\right)\end{array}$ & $\varphi_{1}$ & $\begin{array}{l}\Delta \mathrm{Ks}^{-1} \\
\mathrm{~T} \mathrm{~Pa}^{-1}\end{array}$ & $\Delta \eta \mathrm{cP}$ & $\Delta \mathrm{L}_{\mathrm{f}} \times 10^{5} \mathrm{~m}$ & $\begin{array}{c}\Delta \mathrm{Z} \times 10^{3} \\
\left(\mathrm{Kg} \mathrm{m}^{-2} \mathrm{~s}^{-1}\right)\end{array}$ & $\begin{array}{l}\Delta \mathrm{V}_{\mathrm{f}} \times 10^{-8} \\
\left(\mathrm{~m}^{-3} \mathrm{~mol}^{-1}\right)\end{array}$ \\
\hline 0.0000 & 0.9234 & 0.0000 & 1275 & 0.0000 & 0.0000 & 0.0000 & 0.0000 & 0.0000 & 0.0000 \\
\hline 0.0954 & 0.9773 & -0.2257 & 1212 & 0.0963 & -34.8553 & -0.0371 & 0.4789 & -31.6525 & -0.9080 \\
\hline 0.1935 & 1.0312 & -0.2885 & 1192 & 0.1951 & -58.6987 & -0.0010 & 0.4122 & -26.8480 & -2.1252 \\
\hline 0.2908 & 1.0826 & -0.1537 & 1152 & 0.2930 & -40.9845 & -0.0672 & 0.6837 & -48.4561 & -2.0319 \\
\hline 0.3939 & 1.1368 & -0.0105 & 1100 & 0.3964 & -5.5940 & -0.1003 & 1.1698 & -87.0631 & -2.3998 \\
\hline 0.4931 & 1.1877 & 0.2170 & 1072 & 0.4957 & 4.3230 & -0.1034 & 1.3449 & -104.6740 & -2.7044 \\
\hline 0.6908 & 1.2895 & 0.5596 & 1029 & 0.6930 & 12.5024 & -0.0843 & 1.5557 & -131.4000 & -3.1659 \\
\hline 0.8239 & 1.3598 & 0.6016 & 1007 & 0.8254 & 10.9829 & -0.0263 & 1.6140 & -143.1100 & -4.5454 \\
\hline 0.9190 & 1.4125 & 0.4307 & 994 & 0.9198 & 6.3452 & -0.0376 & 1.6127 & -147.0810 & -3.3494 \\
\hline 1.0000 & 1.4613 & 0.0000 & 1084 & 1.0000 & 0.0000 & 0.0000 & 0.0000 & 0.0000 & 0.0000 \\
\hline
\end{tabular}


$\mathrm{K}$ is Jacobson's constant which is temperature dependent constant but independent of the nature of the liquid.

Viscosity has been calculated using the relation

$$
\eta=(\mathrm{At}-\mathrm{B} / \mathrm{t}) \rho
$$

$\mathrm{A}$ and $\mathrm{B}$ are constants characteristic of viscometer calculated using standard liquids water and nitrobenzene, $t$ time of flow in seconds.

Surianarayana ${ }^{23}$ proposed a relation to calculate free volume

$$
\mathrm{V}_{\mathrm{f}}=\left(\mathrm{M}_{\mathrm{eff}} \mathrm{u} / \mathrm{K} \eta\right)^{3 / 2}
$$

$\mathrm{K}$ is a temperature independent constant which is equal to $4.28 \times 10^{9}$ for all liquids; $M_{\text {eff }}$ is effective molecular weight of the mixture calculated using the relation

$\mathrm{M}_{\text {eff }}=\mathrm{x}_{1} \mathrm{M}_{1}+\mathrm{x}_{2} \mathrm{M}_{2}$. Where $\mathrm{x}_{1}, \mathrm{x}_{2}$, are mole fraction and $\mathrm{M}_{1}, \mathrm{M}_{2}$ molecular weights of the component 1 and 2 , respectively.

Excess volume $\left(\mathrm{V}^{\mathrm{E}}\right)$ has been calculated using the relation

$$
\left.V^{E}=\left(x_{1} M_{1}+x_{2} M_{2}\right) / \rho\right)-\left(x_{1} M_{1} / \rho_{1}\right)-\left(x_{2} M_{2} / \rho_{2}\right)
$$

$\rho_{1}$ and $\rho_{2}$ are densities of pure liquids 1 and $\mathbf{2}$ respectively, $\rho$ is the density of liquid mixture.

Excess adiabatic compressibility $(\Delta \mathrm{Ks})$ has been calculated from the relation

$$
\Delta \mathrm{Ks}=\mathrm{Ks}-\left(\phi_{1} \mathrm{Ks}_{1}+\phi_{2} \mathrm{Ks}_{2}\right)
$$

$\mathrm{Ks}_{1}$ and $\mathrm{Ks}_{2}$ are adiabatic compressibility values of pure liquids and $f_{1}$ and $f_{2}$ are volume fraction for pure liquids calculated by the relation

$$
\phi_{1}=\left(\mathrm{x}_{1} \mathrm{M}_{1} / \rho_{1}\right) /\left(\mathrm{x}_{1} \mathrm{M}_{1} / \rho_{1}+\mathrm{x}_{2} \mathrm{M}_{2} / \rho_{2}\right) \text { and } \phi_{2}=1-\phi_{1}
$$

Excess values of other parameters are calculated using the relation

$$
A^{E}=A_{\exp }-A_{i d}
$$

$A_{i d}=\sum X_{i} A_{i}, X_{i}$ and $A_{i}$ are mole fraction and parameters of the $\mathrm{i}^{\text {th }}$ component liquid.

All the calculated excess values were fitted to Redlich$\mathrm{Kister}^{24}$ type polynomial equation

$$
\mathrm{A}^{\mathrm{E}}=\mathrm{x}_{1} \mathrm{x}_{2}\left[\mathrm{a}+\mathrm{b}\left(\mathrm{x}_{1}-\mathrm{x}_{2}\right)+\mathrm{c}\left(\mathrm{x}_{1}-\mathrm{x}_{2}\right)^{2}\right]
$$

by the method of least squares to derive the adjustable parameters $\mathrm{a}, \mathrm{b}$ and $\mathrm{c}$. From these $\mathrm{a}, \mathrm{b}$ and $\mathrm{c}$ values theoretical values for all excess parameters were calculated and the standard deviation values were calculated using the relation.

$$
\sigma=\left[\left(\mathrm{A}_{\text {exp }}^{\mathrm{E}}-\mathrm{A}_{\text {cal }}^{\mathrm{E}}\right)^{2} /(\mathrm{n}-\mathrm{m})\right]^{1 / 2}
$$

here $\mathrm{n}$ is the number of measurements and $\mathrm{m}$ the number of adjustable parameters. The values of $a, b, c$ and $\sigma$ are given in the Tables $1 \mathrm{c}, 2 \mathrm{c}$ and $3 \mathrm{c}$.

From Tables $1 \mathrm{a}$ and $1 \mathrm{~b}$ it can be seen that all excess values except for viscosity and acoustic impedance are negative at $308.15 \mathrm{~K}$ predicting the presence of strong interaction between morpholine and cyclohexanone. This is due to dipole-dipole, hydrogen bonded network structure formation ${ }^{25,26}$ and electron donor-acceptor complex formation between the mixing liquids, since cyclohexanone is a dipolar molecule and morpholine has two donor centers. Due to these interactions volume contraction takes place and excess volume becomes negative. This nature of interaction is also supported by the negative values of $\Delta \mathrm{Ks}, \Delta \mathrm{L}_{\mathrm{f}}$ and $\Delta \mathrm{V}_{\mathrm{f}}$ values, which indicate the presence of

\begin{tabular}{|c|c|c|c|c|c|c|c|c|c|}
\hline $\mathrm{x}_{1}$ & $\begin{array}{c}\rho \times 10^{3} \\
\left(\mathrm{Kg} \mathrm{m}^{-3}\right)\end{array}$ & $\begin{array}{l}\mathrm{V}^{\mathrm{E}} \times 10^{3} \\
\left(\mathrm{~m}^{3} \mathrm{~mol}^{-1}\right)\end{array}$ & $\mathrm{U}\left(\mathrm{m} \mathrm{s}^{-1}\right)$ & $\varphi_{1}$ & $\begin{array}{l}\Delta \mathrm{Ks} \\
\mathrm{T} \mathrm{Pa}^{-1}\end{array}$ & $\Delta \eta \mathrm{cP}$ & $\Delta \mathrm{L}_{\mathrm{f}} \times 10^{5} \mathrm{~m}$ & $\begin{array}{c}\Delta \mathrm{Z} \times 10^{3} \\
\left(\mathrm{Kg} \mathrm{m}^{-2} \mathrm{~s}^{-1}\right)\end{array}$ & $\begin{array}{l}\Delta \mathrm{V}_{\mathrm{f}} \times 10^{-8} \\
\left(\mathrm{~m}^{-3} \mathrm{~mol}^{-1}\right)\end{array}$ \\
\hline 0.0000 & 0.9312 & 0.0000 & 1358 & 0.0000 & 0.0000 & 0.0000 & 0.0000 & 0.0000 & 0.0000 \\
\hline 0.0956 & 0.9350 & 0.1106 & 1363 & 0.0997 & 0.8387 & 0.1852 & -0.3644 & 2.3055 & -1.6609 \\
\hline 0.1987 & 0.9394 & 0.1903 & 1362 & 0.2062 & 2.9658 & 0.0844 & -0.0612 & -0.7568 & -1.4248 \\
\hline 0.2962 & 0.9437 & 0.2447 & 1361 & 0.3061 & 4.9261 & 0.0197 & 0.2223 & -3.5230 & -1.1903 \\
\hline 0.3947 & 0.9482 & 0.2769 & 1360 & 0.4060 & 6.7833 & -0.0464 & 0.4972 & -6.1046 & -0.6753 \\
\hline 0.4996 & 0.9534 & 0.2594 & 1360 & 0.5113 & 7.6115 & -0.0449 & 0.6413 & -7.2945 & -0.7640 \\
\hline 0.5948 & 0.9583 & 0.2183 & 1362 & 0.6060 & 6.5963 & -0.0505 & 0.5391 & -6.2119 & -0.6134 \\
\hline 0.6974 & 0.9636 & 0.1667 & 1365 & 0.7072 & 4.8302 & -0.0748 & 0.3399 & -4.1832 & 0.0589 \\
\hline 0.8326 & 0.9706 & 0.0892 & 1369 & 0.8390 & 2.5142 & -0.0127 & 0.0813 & -1.3942 & -0.6203 \\
\hline 0.8936 & 0.9737 & 0.0579 & 1371 & 0.8980 & 1.3722 & -0.0430 & 0.0483 & -0.0072 & 0.3509 \\
\hline 1.0000 & 0.9791 & 0.0000 & 1372 & 1.0000 & 0.0000 & 0.0000 & 0.0000 & 0.0000 & 0.0000 \\
\hline
\end{tabular}
compact structure making ability between the liquids. Existence of these specific interaction is also supported by the positive value of $\Delta \mathrm{Z}$ value, since positive values predicts the

\begin{tabular}{|c|c|c|c|c|c|c|c|c|c|}
\hline $\mathrm{x}_{1}$ & $\begin{array}{c}\rho \times 10^{3} \\
\left(\mathrm{Kg} \mathrm{m}^{-3}\right)\end{array}$ & $\begin{array}{l}\mathrm{V}^{\mathrm{E}} \times 10^{3} \\
\left(\mathrm{~m}^{3} \mathrm{~mol}^{-1}\right)\end{array}$ & $\begin{array}{c}\mathrm{U} \\
\left(\mathrm{m} \mathrm{s}^{-1}\right)\end{array}$ & $\varphi_{1}$ & $\begin{array}{l}\Delta \mathrm{Ks} \\
\mathrm{T} \mathrm{Pa}^{-1}\end{array}$ & $\Delta \eta \mathrm{cP}$ & $\Delta \mathrm{L}_{\mathrm{f}} \times 10^{5} \mathrm{~m}$ & $\begin{array}{c}\Delta \mathrm{Z} \times 10^{3} \\
\left(\mathrm{Kg} \mathrm{m}^{-2} \mathrm{~s}^{-1}\right)\end{array}$ & $\begin{array}{l}\Delta \mathrm{V}_{\mathrm{f}} \times 10^{-8} \\
\left(\mathrm{~m}^{-3} \mathrm{~mol}^{-1}\right)\end{array}$ \\
\hline 0.0000 & 0.9234 & 0.0000 & 1275 & 0.0000 & 0.0000 & 0.0000 & 0.0000 & 0.0000 & 0.0000 \\
\hline 0.0956 & 0.9270 & 0.1189 & 1262 & 0.0997 & 23.1437 & 0.1879 & 2.4606 & -18.5910 & -2.4287 \\
\hline 0.1987 & 0.9312 & 0.2051 & 1248 & 0.2062 & 44.2806 & 0.0313 & 5.1173 & -38.3251 & -1.6144 \\
\hline 0.2962 & 0.9354 & 0.2551 & 1236 & 0.3061 & 63.0012 & -0.0172 & 7.4712 & -55.6544 & -1.5477 \\
\hline 0.3947 & 0.9399 & 0.2708 & 1226 & 0.4060 & 79.4717 & -0.0087 & 9.5558 & -70.9538 & -2.1291 \\
\hline 0.4996 & 0.9449 & 0.2579 & 1222 & 0.5113 & 89.2275 & -0.0362 & 10.8381 & -80.8107 & -2.0005 \\
\hline 0.6974 & 0.9547 & 0.1756 & 1252 & 0.7072 & 65.1799 & -0.0607 & 8.0793 & -63.2120 & -1.0309 \\
\hline 0.8326 & 0.9615 & 0.0964 & 1284 & 0.8390 & 38.9693 & -0.0777 & 4.8640 & -39.6637 & 0.4672 \\
\hline 0.8936 & 0.9645 & 0.0657 & 1304 & 0.8980 & 22.7124 & -0.0901 & 2.8148 & -23.6202 & 1.6594 \\
\hline 1.0000 & 0.9698 & 0.0000 & 1334 & 1.0000 & 0.0000 & 0.0000 & 0.0000 & 0.0000 & 0.0000 \\
\hline
\end{tabular}
presence of strong interaction between the liquids. $\Delta \eta$ values are positive over entire mole fraction indicating that flow of

TABLE-3A

THERMODYNAMIC EXCESS PARAMETERS FOR THE BINARY MIXTURE ANISOLE (1)-CYCLOHEXANONE (2) 308.15 K

TABLE-3B

THERMODYNAMIC EXCESS PARAMETERS FOR THE BINARY MIXTURE ANISOLE (1)-CYCLOHEXANONE (2) $318.15 \mathrm{~K}$ 


\begin{tabular}{|c|c|c|c|c|}
\hline \multicolumn{5}{|c|}{$\begin{array}{l}\text { REDLICH-KISTER ADJUSTABLE PARAMETERS AND STANDARD DEVIATION FOR THE BINARY } \\
\text { MIXTURE MORPHOLINE-CYCLOHEXANONE AT 308.15 K. VALUES IN PARENTHESIS ARE FOR } 318.15 \text { K }\end{array}$} \\
\hline & $\mathrm{a}$ & $\mathrm{b}$ & $\mathrm{c}$ & $\sigma$ \\
\hline $\mathrm{V}^{\mathrm{E}}\left(10^{3} \mathrm{Kg} \mathrm{m}^{3}\right)$ & $\begin{array}{l}-0.8576 \\
(-1.4654)\end{array}$ & $\begin{array}{l}-0.9721 \\
(-1.1116)\end{array}$ & $\begin{array}{c}-0.2204 \\
(-0.1888)\end{array}$ & $\begin{array}{c}0.0005 \\
\left(1.0824 \times 10^{-5}\right)\end{array}$ \\
\hline$\Delta \mathrm{Ks}\left(\mathrm{T} \mathrm{Pa}^{-1}\right)$ & $\begin{array}{c}-22.4896 \\
(-188.3660)\end{array}$ & $\begin{array}{c}-2.3187 \\
(126.272)\end{array}$ & $\begin{array}{c}20.2647 \\
(-13.3548)\end{array}$ & $\begin{array}{c}0.0113 \\
(0.0127)\end{array}$ \\
\hline$\Delta \eta(\mathrm{cP})$ & $\begin{array}{c}1.1372 \\
(0.7370)\end{array}$ & $\begin{array}{c}0.1618 \\
(-0.4588)\end{array}$ & $\begin{array}{c}2.1313 \\
(1.9584)\end{array}$ & $\begin{array}{c}0.0008 \\
(0.0007)\end{array}$ \\
\hline$\Delta \mathrm{L}_{\mathrm{f}}(\mathrm{m})$ & $\begin{array}{c}-193897 \\
\left(-2.4791 \times 10^{6}\right)\end{array}$ & $\begin{array}{c}77356 \\
(873369)\end{array}$ & $\begin{array}{c}19842 \\
\left(1.5917 \times 10^{6}\right)\end{array}$ & $\begin{array}{c}0.0023 \\
(0.0112)\end{array}$ \\
\hline$\Delta \mathrm{Z}\left(10^{3} \mathrm{Kg} \mathrm{m}^{-2} \mathrm{~s}^{-1}\right)$ & $\begin{array}{c}8.8372 \\
(179.0630)\end{array}$ & $\begin{array}{c}1.0099 \\
(-78.9857)\end{array}$ & $\begin{array}{c}-0.6622 \\
(-29.0301)\end{array}$ & $\begin{array}{c}0.0188 \\
(0.0144)\end{array}$ \\
\hline$\Delta \mathrm{V}_{\mathrm{f}}\left(\mathrm{m}^{3} \mathrm{~mol}^{-1}\right)$ & $\begin{array}{l}-5.5962 \times 10^{-8} \\
\left(-4.1814 \times 10^{-8}\right)\end{array}$ & $\begin{array}{c}2.1300 \times 10^{-8} \\
\left(4.4462 \times 10^{-8}\right)\end{array}$ & $\begin{array}{c}-1.0122 \times 10^{-7} \\
\left(-1.4400 \times 10^{-7}\right)\end{array}$ & $\begin{array}{c}4.0825 \times 10^{-5} \\
(0.0054)\end{array}$ \\
\hline
\end{tabular}

\begin{tabular}{|c|c|c|c|c|}
\hline \multicolumn{5}{|c|}{$\begin{array}{l}\text { TABLE-2C } \\
\text { REDLICH-KISTER ADJUSTABLE PARAMETERS AND STANDARD DEVIATION FOR THE BINARYMIXTURE } \\
\text { BROMOBENZENE-CYCLOHEXANONE AT } 308.15 \mathrm{~K} \text {. VALUES IN PARENTHESIS ARE FOR } 318.15 \mathrm{~K}\end{array}$} \\
\hline & $\mathrm{a}$ & $\mathrm{b}$ & $\mathrm{c}$ & $\sigma$ \\
\hline $\mathrm{V}^{\mathrm{E}}\left(10^{3} \mathrm{Kg} \mathrm{m}^{3}\right)$ & $\begin{array}{c}0.3802 \\
(0.8892)\end{array}$ & $\begin{array}{c}7.0722 \\
(4.6206)\end{array}$ & $\begin{array}{c}2.8310 \\
(0.6829)\end{array}$ & $\begin{array}{c}0.0040 \\
(0.0003)\end{array}$ \\
\hline$\Delta \mathrm{Ks}\left(\mathrm{T} \mathrm{Pa}^{-1}\right)$ & $\begin{array}{l}-13.3101 \\
(14.4182)\end{array}$ & $\begin{array}{c}216.2390 \\
(318.1760)\end{array}$ & $\begin{array}{c}1.6196 \\
(-367.3560)\end{array}$ & $\begin{array}{c}0.0209 \\
(0.0525)\end{array}$ \\
\hline$\Delta \eta(\mathrm{cP})$ & $\begin{array}{c}-0.4402 \\
(-0.4220)\end{array}$ & $\begin{array}{c}-0.2229 \\
(-0.1798)\end{array}$ & $\begin{array}{c}0.9560 \\
(0.2516)\end{array}$ & $\begin{array}{c}0.0009 \\
(0.0012)\end{array}$ \\
\hline$\Delta \mathrm{L}_{\mathrm{f}}(\mathrm{m})$ & $\begin{array}{c}-252222 \\
\left(4.9115 \times 10^{6}\right)\end{array}$ & $\begin{array}{c}3.0745 \times 10^{6} \\
\left(6.2596 \times 10^{6}\right)\end{array}$ & $\begin{array}{c}-247186 \\
\left(7.5242 \times 10^{6}\right)\end{array}$ & $\begin{array}{c}0.0025 \\
(0.0089)\end{array}$ \\
\hline$\Delta \mathrm{Z}\left(10^{3} \mathrm{Kg} \mathrm{m}^{-2} \mathrm{~s}^{-1}\right)$ & $\begin{array}{c}49.8034 \\
(-379.3750)\end{array}$ & $\begin{array}{l}-350.6840 \\
(-612.8740)\end{array}$ & $\begin{array}{c}-34.0514 \\
(-715.1980)\end{array}$ & $\begin{array}{c}0.0228 \\
(0.7638)\end{array}$ \\
\hline$\Delta \mathrm{V}_{\mathrm{f}}\left(\mathrm{m}^{3} \mathrm{~mol}^{-1}\right)$ & $\begin{array}{l}-5.8140 \times 10^{-8} \\
\left(-9.3789 \times 10^{-8}\right)\end{array}$ & $\begin{array}{c}4.1041 \times 10^{-7} \\
\left(-1.1359 \times 10^{-7}\right) \\
\end{array}$ & $\begin{array}{c}-1.0867 \times 10^{-7} \\
\left(-2.7988 \times 10^{-7}\right)\end{array}$ & $\begin{array}{r}0.0027 \\
(0.0008) \\
\end{array}$ \\
\hline
\end{tabular}

TABLE-3C

REDLICH-KISTER ADJUSTABLE PARAMETERS AND STANDARD DEVIATION FOR THE BINARY

MIXTURE ANISOLE-CYCLOHEXANONE AT 308.15 K. VALUES IN PARENTHESIS ARE FOR 318.15 K

\begin{tabular}{|c|c|c|c|c|}
\hline & $\mathrm{a}$ & $b$ & $\mathrm{c}$ & $\sigma$ \\
\hline $\mathrm{V}^{\mathrm{E}}\left(10^{3} \mathrm{Kg} \mathrm{m}^{3}\right)$ & $\begin{array}{c}1.0275 \\
(1.0265)\end{array}$ & $\begin{array}{c}-0.4610 \\
(-0.4703)\end{array}$ & $\begin{array}{c}-0.2018 \\
(-0.0234)\end{array}$ & $\begin{array}{c}0.0001 \\
\left(4.0824 \times 10^{-5}\right)\end{array}$ \\
\hline$\Delta \mathrm{Ks}\left(\mathrm{T} \mathrm{Pa}^{-1}\right)$ & $\begin{array}{c}29.0526 \\
(343.6160)\end{array}$ & $\begin{array}{c}2.0838 \\
(21.9856)\end{array}$ & $\begin{array}{c}-28.0633 \\
(-164.6420)\end{array}$ & $\begin{array}{c}0.0040 \\
(0.0464)\end{array}$ \\
\hline$\Delta \eta(\mathrm{cP})$ & $\begin{array}{c}-0.2721 \\
(-0.2441)\end{array}$ & $\begin{array}{l}-0.7303 \\
(-0.8094)\end{array}$ & $\begin{array}{c}1.3724 \\
(0.6510)\end{array}$ & $\begin{array}{c}0.0007 \\
(0.0011)\end{array}$ \\
\hline$\Delta \mathrm{L}_{\mathrm{f}}(\mathrm{m})$ & $\begin{array}{c}249637 \\
\left(4.1928 \times 10^{6}\right)\end{array}$ & $\begin{array}{c}111137 \\
(265507)\end{array}$ & $\begin{array}{c}-681557 \\
\left(-2.2134 \times 10^{6}\right)\end{array}$ & $\begin{array}{c}0.0010 \\
(0.0045)\end{array}$ \\
\hline$\Delta \mathrm{Z}\left(10^{3} \mathrm{Kg} \mathrm{m}^{-2} \mathrm{~s}^{-1}\right)$ & $\begin{array}{c}-28.5179 \\
(-314.1320)\end{array}$ & $\begin{array}{c}-7.0168 \\
(-36.9455)\end{array}$ & $\begin{array}{l}59.3328 \\
(139.46)\end{array}$ & $\begin{array}{c}0.0080 \\
(0.0286)\end{array}$ \\
\hline$\Delta \mathrm{V}_{\mathrm{f}}\left(\mathrm{m}^{3} \mathrm{~mol}^{-1}\right)$ & $\begin{array}{l}-2.2325 \times 10^{-8} \\
\left(-6.9025 \times 10^{-8}\right)\end{array}$ & $\begin{array}{c}7.0257 \times 10^{-8} \\
\left(1.2480 \times 10^{-7}\right)\end{array}$ & $\begin{array}{l}-8.7539 \times 10^{-8} \\
\left(6.1257 \times 10^{-8}\right)\end{array}$ & $\begin{array}{c}0.0229 \\
(0.0065)\end{array}$ \\
\hline
\end{tabular}

the liquid mixtures is difficult when compared with pure liquids ${ }^{27}$. The positive value implies the presence of more interaction due to hydrogen bond formation between cyclohexanone and morpholine. As the temperature is raised to $318.15 \mathrm{~K}, \Delta \eta$ values decreases indicating that flow of liquid is made easy as molecules expand with temperature. But at $318.15 \mathrm{~K}$ the negative values of $\mathrm{V}^{\mathrm{E}}, \Delta \mathrm{Ks}, \Delta \mathrm{L}_{\mathrm{f}}$ and $\Delta \mathrm{V}_{\mathrm{f}}$ increases predicting the presence of more interaction between the mixing liquids. This may be due to opening up of agglomerates of morpholine so that more number of cyclohexanone molecules can interact. Similar explanation can be obtained from the $\Delta \mathrm{Z}$ values.
From Tables $2 \mathrm{a}$ and $2 \mathrm{~b}$ for bromobenzene-cyclohexanone mixture the excess values are negative at both the temperatures up to $0.4 / 0.5$ mole fraction values after which the values become positive. But the values of $\Delta \eta$ and $\Delta \mathrm{V}_{\mathrm{f}}$ are negative over entire mole fraction values. $\mathrm{V}^{\mathrm{E}}$ values are negative up to 0.4 mole fraction of bromobenzene predicting more interaction between the liquids through electron-donor acceptor complex formation since bromobenzene is an aromatic liquid with $\pi$ electron cloud. As concentration of bromobenzene is increased it becomes more self agregated ${ }^{28}$ that cyclohexanone find it difficult to interact, so that the nature of interaction then slightly decreases. This nature of interaction is also supported ${ }^{29}$ by the 
$\Delta \mathrm{Ks}, \Delta \mathrm{L}_{\mathrm{f}}$ and $\Delta \mathrm{Z}$ values. $\Delta \eta$ values are low negative predicting easy flow of the mixtures compared to pure liquids. Increase of temperature also has the same effect on the excess values and the values of parameters have increased showing more interaction between the mixing liquids.

From Tables $3 \mathrm{a}$ and $3 \mathrm{~b}$ for anisole-cyclohexanone binary mixture $\mathrm{V}^{\mathrm{E}}$ and $\Delta \mathrm{Ks}$ values are positive predicting expansion of volume due to more liquid order breaking effect than association type of interaction. These values indicate non-specific physical interaction and unfavorable interaction between unlike molecules $i e$; dispersion type of force ${ }^{30}$ prevails though both are polar liquids. At $318.15 \mathrm{~K}$ also same nature of interaction is predicted which can be seen from the positive values of excess parameters. The excess values of $\Delta \mathrm{L}_{\mathrm{f}}, \Delta \mathrm{Z}$ and $\Delta \mathrm{V}_{\mathrm{f}}$ predict the same nature of interaction at both temperatures. $\Delta \eta$ values are positive up to 0.3 mole fraction of anisole and then the value becomes negative, same effect is seen at higher temperature. This sign of $\Delta \eta$ predicts that flow of mixture is difficult at lower concentration of anisole and then the rate of flow increases as the concentration of anisole is increased.

\section{Conclusion}

From the density, viscosity and ultrasonic velocity of the binary systems studied at $308.15 \mathrm{~K}$ and $318.15 \mathrm{~K}$ it can be predicted that there is specific type of interaction between cyclohexanone and morpholine, bromobenzene and nonspecific type of interaction between cyclohexanone and anisole. $\Delta \mathrm{Ks}$ and $\mathrm{V}^{\mathrm{E}}$ shows a trend morpholine $>$ bromobenzene $>$ anisole and $\Delta \eta$ shows a reverse trend on the nature of interactions with cyclohexanone.

\section{REFERENCES}

1. X. Rosario Rajkumar, K.V. Raman and S.J. Arulraj, Acta Ciencia Indica, 7c, 96 (1981)

2. G.V. Rama Rao, A. Viswanath Sarma and C. Rambabu, Indian J. Chem., 43A, 2518 (2004).

3. S. Acharya, B.K. Das and G.C. Mohanty, Indian J. Phys., 83, 185 (2009).

4. E.J. Gonzalez, B. Gonzalez and E.A. Macedo, J. Chem. Eng. Data, 58, 1440 (2013).

5. A.R. Mahajan and S.R. Mirgane, J. Solution Chem., 42, 1146 (2013).

6. D.R. Cova, J. Chem. Eng. Data, 5, 282 (1960).

7. K.P.C. Rao and K.S. Reddy, Can. J. Chem. Eng., 66, 474 (1988).

8. N.G. Tsierkezos, I.E. Molinou and A.C. Filippou, J. Solution Chem., 34, 1371 (2005).

9. M.I. Aralaguppi, C.V. Jadar and T.M. Aminabhavi, J. Chem. Eng. Data, 44, 435 (1999).

10. A.M. Awwad, E.I. Allos and S.R. Salman, J. Chem. Eng. Data, 33, 265 (1988).

11. G. Parthipan and T. Thenappan, J. Mol. Liq., 138, 20 (2008).

12. G.V. RamaRao, Indian J. Pure Appl. Phys., 42, 820 (2004).

13. G.K. Raman and P.R. Naidu, Proc. Math. Sci., 77, 263 (1973).

14. S. Varshnay and M. Singh, J. Chem. Eng. Data, 51, 1136 (2006).

15. M.B. Shiflett, A.M.S. Niehaus and A. Yokozeki, J. Chem. Eng. Data, 54, 2090 (2009).

16. S.S. Joshi and T.M. Aminabhavi, Fluid Phase Equb., 60, 319 (1990).

17. J. Nath, J. Chem. Thermodyn., 28, 481 (1996).

18. V. Mutalik, L.S. Manjeshwar, M. Sairam and T.M. Aminabhavi, J. Chem. Thermodyn., 38, 1620 (2006).

19. B.S. Furniss, A.J. Hannaford, P.W.G. Smith and A.R. Tatchell, Vogel's Text book of Practical Organic Chemistry, edn 5, Ch. 2, p. 165 Longman, London (1989).

20. A. Rose Venis and X. Rosario Rajkumar, Oriental J. Chem., 27, 105 (2011).

21. P.S. Nikam and M. Hasan, Indian J. Pure Appl. Phys., 24, 502 (1986).

22. B. Jacobson, J. Chem. Phys., 20, 927 (1952).

23. C.V. Suryanarayana, J. Acoust. Soc. India, 7, 107 (1976).

24. O. Redlich and A.T. Kister, Ind. Eng. Chem., 40, 345 (1948).

25. R.J. Sengwa, V. Khatri and S. Sankhla, Indian J. Chem., 48A, 512 (2009).

26. A. Rose Venis and X. Rosario Rajkumar, Asian J. Chem., 24, 1195 (2012).

27. P. Rathore and M. Singh, Indian J. Chem., 45A, 2650 (2006).

28. G. Nath, S. Sahu and R. Paikaray, Indian J. Phys, 83, 429 (2009).

29. P.S. Nikam, V.M. Kapade and M. Hasan, Indian J. Pure Appl. Phys., 38, 170 (2000).

30. R. Mehra, A. Gupta and R. Israni, Indian J. Chem., 40A, 505 (2001). 